\title{
Task-specific serial position effects in comparisons of multiletter strings
}

\author{
ROBERT W. PROCTOR \\ Auburn University, Auburn, Alabama \\ and \\ ALICE F. HEALY \\ University of Colorado, Boulder, Colorado
}

\begin{abstract}
Several recent studies of multiletter matching have included pairs in which the two letter strings have the same letters but in different orders. The latency of responses and the error rates to these rearranged pairs vary as a function of the total number of positions by which the letters in one string are displaced in the other. When order is relevant, and the rearranged pairs are classified as "different" (the order task), both response measures decrease as displacement increases. Similar, but mirror-image, displacement functions are obtained when order is irrelevant and the rearranged pairs are classified as "same" (the item task). The present experiments compared displacement with other measures of permutation and included a systematic examination of serial-position functions for the two tasks. Displacement, rather than the other measures of permutation, best predicted performance on both the order and item tasks, and the order task consistently showed a greater left-to-right weighting of serial positions than did the item task. Thus, even though the displacement functions are qualitatively similar for the two tasks, the difference in serialposition functions indicates that subjects exert some strategic control over the comparison of multiletter strings.
\end{abstract}

In studies of multiletter matching, subjects judge pairs of letter strings as being either "same" or "different." Traditionally, such studies have used a procedure in which the two strings that form a same pair are identical (i.e., the strings contain the same letters in the same order), whereas the strings that form a different pair mismatch at one or more positions (e.g., Bamber, 1969; Proctor, Rao, \& Hurst, 1984). In other words, subjects must discriminate identical pairs (e.g., ABC-ABC) from replacement pairs in which one or more letters from one string have been replaced to form the other string (e.g., $\mathrm{ABC}-\mathrm{ABX}$ ).

The traditional procedure has been modified in recent studies to include rearranged pairs in which the two strings within a pair have the same letters, but in different orders (e.g., ABC-ACB; Angiolillo-Bent \& Rips, 1982; Proctor \& Healy, 1985; Ratcliff, 1981; Ratcliff \& Hacker,

This research was supported by National Science Foundation Grant BNS80-25020 to the University of Colorado; by BRSG Grant RR0701316 awarded to the University of Colorado by the Biomedical Research Support Grant Program, Division of Resources, National Institute of Health; and by research grants-in-aid from the University of Colorado Council on Research and Creative Work and from the Auburn University Office of Research. We would like to thank James McAlarney III for programming Experiment 1; Judson Butler, Steve Monda, Hunter Peak, and Mike Thompson for their assistance in conducting Experiment 1; and Grant Sinclair, David Fendrich, Barbara White, and Antoinette Gesi for their assistance in designing, conducting, and analyz ing Experiment 2. Reprint requests should be sent to Robert W. Proctor, Department of Psychology, Auburn University, Auburn, AL 36849.
1981). The rearranged pairs can be classified as either "different" or "same," depending on whether order is relevant or irrelevant to the decision (i.e., whether pairs are to be classified as "same" only if the two strings have the same letters in the same order, or if the strings have the same letters in any order). When order is relevant (henceforth referred to as the order task), subjects must discriminate identical pairs from both rearranged and replacement pairs. Thus, between-position comparisons (i.e., the comparisons that involve letters occurring at noncorresponding serial positions in the two strings) are extraneous to the "same"/"different" decision. However, when order is irrelevant (henceforth referred to as the item task), the between-position comparisons are pertinent, because the rearranged, as well as the identical, pairs must be discriminated from the replacement pairs.

Several important findings have emerged from the studies that have used the order and/or item tasks. First, reaction times (RTs) and errors in classifying the rearranged pairs as "same" in the item task are an increasing function of the total number of positions by which the letters in one string are displaced to form the other (i.e., the displacement count; Angiolillo-Bent \& Rips, 1982; Proctor \& Healy, 1985), indicating that item information cannot be accessed independently of order information when order is irrelevant to the decision. Second, RTs and errors in classifying the rearranged pairs as "different" in the order task are a decreasing function of the displacement count (Proctor \& Healy, 1985; Ratcliff, 1981; Rat- 
cliff \& Hacker, 1981), demonstrating that extraneous between-position comparisons cannot be avoided when order is relevant to the decision. Third, with the exception of direction, the displacement effects for the order and item tasks are qualitatively and quantitatively similar (Proctor \& Healy, 1985), suggesting that the comparison operations are similar for the two tasks.

The similar displacement effects for the order and item tasks are explained best by a variation of a positionsensitive (or distance-sensitive) comparison model proposed initially by Angiolillo-Bent and Rips (1982) for the item task. In their original model, each letter in one string is compared in parallel to each letter in the other string, with the time to complete any given comparison being an increasing function of the relative positional separation of the two letters in the respective strings. Thus, for example, within-position comparisons (i.e., the comparisons between letters in corresponding positions) should be completed more rapidly than between-position comparisons. Angiolillo-Bent and Rips (1982) concluded that independent, self-terminating decisions were made for each comparison. The assumption that such a comparison process also is used for the order task leads to the prediction that the effects of displacement should be attenuated in that situation, because the rapid discovery of a within-position mismatch could terminate the comparison process before the slower between-position comparisons were completed. Therefore, the mirror-image effects for the order and item tasks obtained by Proctor and Healy (1985) suggest that the "same"/" different" decision is based not on independent decisions, but on pooled information from the component comparisons (e.g., Ratcliff, 1981). Moreover, because the rearrangement effects are of similar magnitude in the order task, for which the between-position comparisons are extraneous, and in the item task, for which the between-position comparisons are required, the comparisons apparently are an unavoidable consequence of the internal structure of the string representations.

Although Proctor and Healy (1985) stressed the similarity of the comparison operations for the order and item tasks, their data included results suggesting that taskspecific processes might also be involved. More precisely, a comparison between rearranged pairs of three-letter strings that had equivalent displacement counts but that differed in whether the two strings matched in the leftmost or rightmost position suggested that the left position may be weighted relatively more for the order task than for the item task. However, this possible difference between the two tasks was downplayed by Proctor and Healy because it involved only a single comparison and may have resulted either from differences in the stimulus sets used for the two tasks or from differences in the response criteria employed (see the introduction to the present Experiment 2). Because any differences in the serial-position functions for the two tasks would be a strong indication of processing differences, the present study provided an examination of the functions that was more thorough and better controlled in three respects.
First, the use of four-letter strings, rather than three-letter strings, permitted more systematic evaluations of the serial-position effects for the rearranged pairs. Second, the use of the same stimulus sets in the two tasks, rather than different sets, and the systematic manipulation of response criteria permitted the testing of two possible explanations for the different serial-position functions. Third, the increase in the number and type of replacement pairs in the order task permitted a comparison of the serial-position functions in the two tasks for the replacement pairs as well as for the rearranged pairs.

Proctor and Healy's (1985) demonstration that the RTs and errors for both the order and item task were a function of displacement count involved a relatively weak manipulation of displacement. That is, the three-letter strings that were used allow only two levels of displacement to be compared. Because these two levels are confounded closely with characteristics of the rearranged pairs that alternatives to the position-sensitive comparison model predict to be important (see the introduction to the present Experiment 1), the results could be a function of one of these other characteristics. Thus, an additional purpose of the present study was to perform a stronger manipulation of displacement count to determine more conclusively whether displacement, rather than some other measure of permutation, is the crucial factor.

\section{EXPERIMENT 1}

Several characteristics of the rearranged pairs are confounded with total displacement for the three-letter strings used by Proctor and Healy (1985). These characteristics include (1) the first position, proceeding in a left-to-right order, in which a within-position mismatch occurs; (2) the total number of within-position mismatches (or matches); and (3) the minimum number of perturbations (or transpositions of neighboring letter pairs) necessary for the representations of the two strings to have the same order. These characteristics are predicted to be crucial by models that hypothesize, respectively, (1) only an analytic, leftto-right serial scanning process (e.g., Krueger, 1984); (2) a holistic or analytic parallel comparison process without between-position comparisons (e.g., Krueger, 1978); and (3) perturbations of order in perception and memory (e.g., Lee \& Estes, 1981).

Experiment 1 used four-letter strings, rather than the three-letter strings used previously, with half of the subjects performing the order task and half the item task. The use of four-letter strings allows a more stringent test of the position-sensitive comparison model's prediction that total displacement is the primary determinant of difficulty for the rearranged pairs. This more stringent test occurs because there are 23 possible rearranged permutations of four-letter strings that have four different displacement counts $(2,4,6$, or 8 positions), rather than only the 5 permutations with two displacement counts ( 2 or 4 positions) that exist for three-letter strings. More importantly, as shown in Table 1, the three characteristics noted above 
Table 1

Displacement Count, Position of Initial Difference, Number of Within-Position Mismatches, and Minimum Number of Perturbations for the Four-Letter Strings Used in Experiment 1

\begin{tabular}{ccccc}
\hline $\begin{array}{c}\text { Base } \\
\text { Permutation }\end{array}$ & $\begin{array}{c}\text { Displacement } \\
\text { Count }\end{array}$ & $\begin{array}{c}\text { Position of } \\
\text { Initial } \\
\text { Difference }\end{array}$ & $\begin{array}{c}\text { Number of } \\
\text { Within-Position } \\
\text { Mismatches }\end{array}$ & $\begin{array}{c}\text { Minimum Number } \\
\text { of Perturbations }\end{array}$ \\
\hline ABCD & 0 & 0 & 0 & 0 \\
ABDC & 2 & 3 & 2 & 1 \\
ACBD & 2 & 2 & 2 & 1 \\
BACD & 2 & 1 & 2 & 1 \\
ACDB & 4 & 2 & 3 & 2 \\
ADBC & 4 & 2 & 3 & 3 \\
ADCB & 4 & 2 & 2 & 2 \\
BADC & 4 & 1 & 4 & 2 \\
BCAD & 4 & 1 & 3 & 2 \\
CABD & 4 & 1 & 3 & 3 \\
CBAD & 4 & 1 & 2 & 3 \\
BCDA & 6 & 1 & 4 & 4 \\
BDAC & 6 & 1 & 4 & 3 \\
BDCA & 6 & 1 & 3 & 4 \\
CABD & 6 & 1 & 4 & 3 \\
DABC & 6 & 1 & 3 & 4 \\
CBDA & 6 & 1 & 4 & 4 \\
DACB & 6 & 1 & 3 & 5 \\
DBAC & 6 & 1 & 3 & 4 \\
DBCA & 6 & 1 & 2 & 5 \\
CDAB & 8 & 1 & 4 & 5 \\
CDBA & 8 & 1 & 4 & 6 \\
DCAB & 8 & 1 & 4 & 3 \\
DCBA & 8 & 1 & 4 & \\
\hline
\end{tabular}

*From left to right.

that are confounded with displacement count for threeletter strings do not vary systematically with displacement for four-letter strings. Thus, the four-letter strings should show more conclusively whether total displacement is crucial.

Increasing the string length from three to four letters also allows a more precise evaluation of the possibility that the order task involves a greater left-to-right weighting of serial positions than does the item task. The major reason for this is that the four-letter strings contain more permutations, across a variety of displacement counts, that differ in terms of critical serial positions (e.g., whereas only one rearranged permutation has a match in the leftmost position for three-letter strings, five permutations do for four-letter strings). The greater variety of permutations allows evaluation of alternative explanations of the apparent difference in weightings for the two tasks, as well as determination of whether the difference is limited to the leftmost position. In addition, the four-letter strings permit more positions of replacement for the replacement pairs, thus providing further evidence regarding the extent of differences in the serial-position weightings for the two tasks.

Half of the subjects who used each decision rule were tested with simultaneous presentation of the two strings within each pair, whereas half were tested with successive presentation. Proctor and Healy (1985) found that the permutation effects for rearranged pairs were qualitatively similar for simultaneous and successive presentation, with only slight quantitative differences in the ef- fects. This outcome is interesting because recent studies that required subjects to discriminate between only identical and replacement pairs have suggested that differences exist in the processing of simultaneously and successively presented strings (Krueger, 1984; Proctor et al., 1984). However, such differences between the two presentation methods have been most apparent for strings of more than three letters. Therefore, the four-letter strings used in the present experiment provide a greater opportunity for differences between simultaneous and successive presentation to appear.

\section{Method}

\section{Subjects and Design}

The subjects were 64 students enrolled in an introductory psychology course at Auburn University. Each subject participated in two sessions for extra credit toward his/her course grade. Type of matching rule (order, item) and method of presentation (simultaneous, successive) were varied between subjects, making four groups of 16 subjects each. Type of pair (described below) was manipulated within subjects.

\section{Apparatus}

Stimuli were presented in white on the dark display screen of a Radio Shack TRS-80 Model 4 microcomputer, which has a P-4 phosphor that decays to $1 \%$ of its maximum intensity in $60 \mathrm{msec}$. The stimuli were composed of letters from the standard character set of the computer. Timing was controlled by the computer, with RTs recorded to the nearest millisecond.

Subjects responded by pressing one of two response keys on the computer's keyboard. Half of the subjects in each condition responded "same"' by pressing the Z key (the leftmost key on the bottom row) with the left index finger and "different" by pressing 
the ? key (the rightmost key on the bottom row) with the right index finger. For the other half of the subjects the assignments were reversed.

\section{Stimuli}

The stimuli were pairs of four-letter strings composed from uppercase versions of all consonants of the alphabet (excluding $\mathrm{Y}$ ), with all letters used approximately equally often. No letters were repeated within a string or on successive trials. The two strings in each pair were centered immediately above and immediately below a fixation point that consisted of a pair of asterisks aligned with the middle two positions of the strings. From an approximate viewing distance of $50 \mathrm{~cm}$, the separation between the top and bottom strings was $.80^{\circ}$. Each letter was $.23^{\circ}$ wide and $.57^{\circ}$ high, with $.06^{\circ}$ separation between letters. Thus, each four-letter string was $1.10^{\circ}$ wide.

Two different types of lists were constructed, one for each of the tasks (order and item). The lists consisted of blocks of 96 pairs, half of which were same pairs in the appropriate task (i.e., pairs for which the correct response was "same") and half of which were different pairs (i.e., pairs for which the correct response was "different"). The types of pairs employed are indicated best by using $A B C D$ to designate the first string, then referring to the pair by using letters to reflect the relation of the second string to the first (this terminology also was used by Angiolillo-Bent \& Rips, 1982, and Proctor \& Healy, 1985). Table 2 includes a useful summary of the block compositions for each task, which were as follows. For the order lists, all 48 same pairs within each block were of the $A B C D$ type. For the item lists, only two same pairs were of the $A B C D$ type; the remaining 46 same pairs in the block were divided equally among the 23 possible permutations of the four letters: $\mathrm{ABDC}, \mathrm{ACBD}$, and BACD (with a displacement count of 2);
ACDB, ADBC, ADCB, BADC, BCAD, CABD, and CBAD (displacement count 4); BCDA, BDAC, BDCA, CADB, CBDA, DABC, DACB, DBAC, and DBCA (displacement count 6); and CDAB, CDBA, DCAB, and DCBA (displacement count 8). Thus, each of the 24 types of same pairs occurred two times within each block in the item list.

The 23 rearranged pairs also occurred two times each in the order list, but as different pairs. The remaining 2 different pairs for this list were selected from replacement pairs of type XBCD, AXCD, $A B X D$, and $A B C X$, in which a new letter was substituted in the second string in place of one of the letters from the first string without rearranging the order. Across blocks, each position of replacement was used equally often for these pairs. Different pairs for the item list included the instances of these 4 replacement pairs, along with similar replacement pairs for each of the rearranged orders (e.g., DXCB, DAXB). Within each block of the item list, there were 48 replacement pairs, with 2 derived from each of the 24 letter permutations. Across blocks, each position of replacement was used equally often.

Two sets of four blocks were constructed for both the order and item lists. The lists of each type were coupled in such a way that the equivalent pair types in each list were in fact the same stimuli (i.e., they were composed of the same letters). Moreover, the positions of these pairs of letter strings were the same in the two list types. This coupling was accomplished by constructing the blocks of 96 trials for the item lists, then constructing the corresponding order lists by replacing the replacement pairs (except those constructed from the ABCD base strings) from each block in the item lists with identical pairs of the ABCD type. With this construction, the sequence of same and different pairs necessarily differed between the coupled lists, but for both the order and item lists each block of 96 trials included 48 same and 48 different pairs.

Table 2

Number of Pairs of Each Type in Each Block of 96 Trials for the Respective Tasks in Experiment 1

\begin{tabular}{|c|c|c|c|c|c|c|}
\hline \multirow{4}{*}{$\begin{array}{c}\text { Base } \\
\text { Permutation }\end{array}$} & \multicolumn{6}{|c|}{ Task } \\
\hline & \multicolumn{3}{|c|}{ Order } & \multicolumn{3}{|c|}{ Item } \\
\hline & \multirow{2}{*}{$\frac{\text { Same }}{\text { Identical }}$} & \multicolumn{2}{|c|}{ Different } & \multicolumn{2}{|c|}{ Same } & \multirow{2}{*}{$\frac{\text { Different }}{\text { Replacement* }}$} \\
\hline & & Rearranged & Replacement* & Identical & Rearranged & \\
\hline ABCD & 48 & - & 2 & 2 & - & 2 \\
\hline ABDC & - & 2 & - & - & 2 & 2 \\
\hline ACBD & - & 2 & - & - & 2 & 2 \\
\hline BACD & - & 2 & - & - & 2 & 2 \\
\hline $\mathrm{ACDB}$ & - & 2 & - & - & 2 & 2 \\
\hline ADBC & - & 2 & - & - & 2 & 2 \\
\hline ADCB & - & 2 & - & - & 2 & 2 \\
\hline BADC & - & 2 & - & - & 2 & 2 \\
\hline BCAD & - & 2 & - & - & 2 & 2 \\
\hline CABD & - & 2 & - & - & 2 & 2 \\
\hline CBAD & - & 2 & - & - & 2 & 2 \\
\hline $\mathrm{BCDA}$ & - & 2 & - & - & 2 & 2 \\
\hline BDAC & - & 2 & - & - & 2 & 2 \\
\hline BDCA & - & 2 & - & - & 2 & 2 \\
\hline CADB & - & 2 & - & - & 2 & 2 \\
\hline CBDA & - & 2 & - & - & 2 & 2 \\
\hline DABC & - & 2 & - & - & 2 & 2 \\
\hline DACB & - & 2 & - & - & 2 & 2 \\
\hline DBAC & - & 2 & - & - & 2 & 2 \\
\hline DBCA & - & 2 & - & - & 2 & 2 \\
\hline CDAB & - & 2 & - & - & 2 & 2 \\
\hline CDBA & - & 2 & - & - & 2 & 2 \\
\hline DCAB & - & 2 & - & - & 2 & 2 \\
\hline DCBA & - & 2 & - & - & 2 & 2 \\
\hline
\end{tabular}

*Within a block, the two replacement pairs for each permutation had different positions of replacement (Positions 1-4); across blocks, all positions were sampled equally often. 
Table 3

Reaction Times (in Milliseconds) and Percentages of Errors for Position-Identical and Rearranged Letter Pairs as a Function of Displacement Count, Permutation, Task, and Method of Presentation in Experiment 1

\begin{tabular}{|c|c|c|c|c|c|c|c|c|}
\hline \multirow{3}{*}{$\begin{array}{c}\text { Base } \\
\text { Permutation* }\end{array}$} & \multicolumn{4}{|c|}{ Reaction Time } & \multicolumn{4}{|c|}{ Percentage of Errors } \\
\hline & \multicolumn{2}{|c|}{ Order Task } & \multicolumn{2}{|c|}{ Item Task } & \multicolumn{2}{|c|}{ Order Task } & \multicolumn{2}{|c|}{ Item Task } \\
\hline & Simultaneous & Successive & Simultaneous & Successive & Simultaneous & Successive & Simultaneous & Successive \\
\hline $\operatorname{ABCD}(0)$ & 1078 & 745 & 1293 & 845 & 3.1 & 4.7 & 2.0 & 2.3 \\
\hline$A B D C(2)$ & 1263 & 1052 & 1507 & 1013 & 23.1 & 36.2 & 7.0 & 7.8 \\
\hline $\mathrm{ACBD}(2)$ & 1134 & 933 & 1523 & 943 & 18.4 & 33.2 & 4.7 & 6.3 \\
\hline $\mathrm{BACD}(2)$ & 825 & 789 & 1703 & 1049 & 6.3 & 19.1 & 7.4 & 8.6 \\
\hline $\mathrm{ACDB}(4)$ & 1016 & 926 & 1637 & 1103 & 2.3 & 16.4 & 7.0 & 6.3 \\
\hline ADBC(4) & 1015 & 823 & 1689 & 1127 & 5.1 & 13.7 & 6.3 & 9.0 \\
\hline $\operatorname{ADCB}(4)$ & 1081 & 823 & 1737 & 1191 & 5.9 & 9.4 & 7.0 & 8.6 \\
\hline BADC(4) & 804 & 721 & 1886 & 1177 & 1.6 & 2.3 & 7.8 & 14.4 \\
\hline $\mathrm{BCAD}(4)$ & 826 & 772 & 1704 & 1153 & 3.5 & 5.1 & 6.6 & 9.8 \\
\hline CABD(4) & 791 & 709 & 1761 & 1098 & 3.5 & 4.3 & 3.5 & 7.8 \\
\hline CBAD(4) & 806 & 746 & 1753 & 1131 & 5.5 & 8.6 & 5.5 & 10.6 \\
\hline BCDA(6) & 734 & 684 & 1825 & 1292 & 2.0 & 3.5 & 8.2 & 9.0 \\
\hline BDAC(6) & 739 & 699 & 2023 & 1270 & 1.2 & 8.6 & 9.8 & 14.8 \\
\hline BDCA(6) & 790 & 692 & 1847 & 1361 & 1.2 & 1.6 & 8.2 & 12.9 \\
\hline $\mathrm{CADB}(6)$ & 753 & 652 & 1794 & 1235 & 0.8 & 1.6 & 10.2 & 10.2 \\
\hline CBDA(6) & 757 & 713 & 1817 & 1250 & 2.0 & 3.5 & 6.3 & 16.8 \\
\hline DABC(6) & 732 & 686 & 1833 & 1248 & 2.0 & 2.3 & 5.5 & 16.4 \\
\hline $\operatorname{DACB}(6)$ & 769 & 675 & 1926 & 1315 & 1.2 & 1.2 & 9.4 & 14.8 \\
\hline DBAC (6) & 768 & 679 & 1911 & 1341 & 2.0 & 2.7 & 13.7 & 16.4 \\
\hline DBCA(6) & 745 & 665 & 1932 & 1242 & 1.6 & 7.8 & 6.3 & 20.6 \\
\hline $\mathrm{CDAB}(8)$ & 718 & 693 & 1794 & 1215 & 2.3 & 3.1 & 12.5 & 16.4 \\
\hline CDBA(8) & 711 & 650 & 1847 & 1233 & 1.6 & 0.4 & 6.6 & 18.4 \\
\hline $\operatorname{DCAB}(8)$ & 707 & 650 & 1826 & 1293 & 0.4 & 2.7 & 9.0 & 13.7 \\
\hline DCBA(8) & 746 & 674 & 1856 & 1251 & 3.5 & 7.8 & 7.0 & 12.5 \\
\hline
\end{tabular}

*Displacement count is in parentheses.

\section{Procedure}

Subjects who performed the item task were instructed to respond "same" if the two strings contained the same letters, regardless of whether the order was the same. Subjects who performed the order task were told to respond "same" only if the two strings had the same letters in the same order. The instructions stressed responding rapidly without making too many errors.

At the start of each session, a practice block of 16 trials was presented. This block was followed by four test blocks of 96 trials each. For each condition, the order of the specific blocks was counterbalanced across subjects by using four Latin squares that comprised 16 of the 24 possible permutations of order. The two sets of blocks also were counterbalanced across sessions. Each block was initiated by the subject once a prompt had been displayed on the screen.

For each subject, the pairs were presented either all simultaneously or all successively. The general procedure was as follows. The fixation asterisks occurred as a warning signal for $1 \mathrm{sec}$. At their offset, a pair of letter strings was presented. For the simultaneous condition, both strings appeared at the same time and remained in view until the subject responded. For the successive condition, the string above fixation was presented for $500 \mathrm{msec}$, followed by a blank interval of $500 \mathrm{msec}$, and then presentation of the second string until the subject responded. For both methods of presentation, the fixation asterisks reappeared as a warning signal 500 msec after the response. $R T$ was measured from the onset of the second string.

\section{Results and Discussion}

Mean RTs for correct responses and percentages of errors were obtained for each subject as a function of pair type. The values for the identical pairs in the order task were computed on the subset of these pairs that also was in the item task. The data are summarized in Table 3 for the identical and rearranged pairs and in Tables 4 and 5 , respectively, for the replacement pairs in the order and item tasks. For the primary data analyses, the identical, rearranged, and replacement pairs were analyzed separately. Both tasks (order and item) were analyzed together for the former two pair types, but separately for the latter pair type. Distinct analyses of each task were required for the replacement pairs because the pairs used in the two tasks were different. Except where noted, the RT and error data yielded consistent results (i.e., RT and percentage of errors were positively correlated).

\section{Method of Presentation and Task}

The main effects for method of presentation and task were not of primary concern in the present investigation. However, the effects were similar to those reported by Proctor and Healy (1985) for three-letter strings. ${ }^{1}$ Responses were faster with successive presentation than with simultaneous presentation for all pair types $[F \mathrm{~s} \geq 12.0$, ps $<.005]$, with only the rearranged pairs showing significantly more errors with successive presen-

Table 4

Reaction Times (RT, in Milliseconds) and Percentages of Errors (\%E) for Replacement Pairs in the Order Task for Experiment 1, as a Function of Position of Replacement

\begin{tabular}{|c|c|c|c|c|c|c|c|c|}
\hline \multirow{3}{*}{$\begin{array}{c}\text { Method of } \\
\text { Presentation }\end{array}$} & \multicolumn{8}{|c|}{ Position of Replacement } \\
\hline & \multicolumn{2}{|c|}{$\begin{array}{c}1 \\
(\mathrm{XBCD})\end{array}$} & \multicolumn{2}{|c|}{$\begin{array}{c}2 \\
\text { (AXCD) }\end{array}$} & \multicolumn{2}{|c|}{$\begin{array}{c}3 \\
(\mathrm{ABXD})\end{array}$} & \multicolumn{2}{|c|}{$\begin{array}{c}4 \\
(\mathrm{ABCX})\end{array}$} \\
\hline & RT & $\overline{\mathscr{F E}}$ & RT & $\% \mathrm{E}$ & $\overline{\mathrm{RT}}$ & $\% \mathrm{E}$ & RT & $\% \mathrm{E}$ \\
\hline $\begin{array}{l}\text { Simultaneous } \\
\text { Successive }\end{array}$ & $\begin{array}{l}784 \\
759\end{array}$ & $\begin{array}{r}4.7 \\
11.0\end{array}$ & $\begin{array}{r}1046 \\
769\end{array}$ & $\begin{array}{l}17.2 \\
11.0\end{array}$ & $\begin{array}{r}1127 \\
813\end{array}$ & $\begin{array}{r}14.1 \\
6.3\end{array}$ & $\begin{array}{r}1331 \\
894\end{array}$ & $\begin{array}{l}25.0 \\
23.5\end{array}$ \\
\hline
\end{tabular}


Table 5

Reaction Times (in Milliseconds) and Percentages of Errors for Replacement Pairs in the Item Task for Experiment 1

\begin{tabular}{|c|c|c|c|c|c|c|c|c|c|}
\hline \multirow{3}{*}{$\begin{array}{c}\text { Base } \\
\text { Displacement } \\
\text { Count } \\
\end{array}$} & \multirow{3}{*}{$\begin{array}{c}\text { Base } \\
\text { Permutation }\end{array}$} & \multicolumn{8}{|c|}{ Method and Position of Replacement } \\
\hline & & \multicolumn{4}{|c|}{ Simultaneous } & \multicolumn{4}{|c|}{ Successive } \\
\hline & & 1 & 2 & 3 & 4 & 1 & 2 & 3 & 4 \\
\hline \multicolumn{10}{|c|}{ Reaction Times } \\
\hline 0 & ABCD & 1365 & 1774 & 1520 & 1655 & 979 & 1153 & 1101 & 1072 \\
\hline 2 & $\begin{array}{l}\text { ABDC } \\
\text { ACBD } \\
\text { BACD }\end{array}$ & $\begin{array}{l}1802 \\
1736 \\
1624\end{array}$ & $\begin{array}{l}1601 \\
1672 \\
1745\end{array}$ & $\begin{array}{l}1457 \\
1837 \\
1775\end{array}$ & $\begin{array}{l}1661 \\
1645 \\
1960\end{array}$ & $\begin{array}{l}1203 \\
1168 \\
1135\end{array}$ & $\begin{array}{l}1022 \\
1112 \\
1078\end{array}$ & $\begin{array}{r}973 \\
1136 \\
1094\end{array}$ & $\begin{array}{l}1200 \\
1138 \\
1200\end{array}$ \\
\hline 4 & $\begin{array}{l}\text { ACDB } \\
\text { ADBC } \\
\text { ADCB } \\
\text { BADC } \\
\text { BCAD } \\
\text { CABD } \\
\text { CBAD }\end{array}$ & $\begin{array}{l}2006 \\
1679 \\
1545 \\
1731 \\
1971 \\
1930 \\
1692\end{array}$ & $\begin{array}{l}1817 \\
2023 \\
1501 \\
1782 \\
1551 \\
1895 \\
1528\end{array}$ & $\begin{array}{l}1724 \\
1611 \\
1654 \\
1949 \\
1653 \\
1515 \\
1474\end{array}$ & $\begin{array}{l}1632 \\
1554 \\
1551 \\
1630 \\
1840 \\
1621 \\
1632\end{array}$ & $\begin{array}{r}1220 \\
1096 \\
1067 \\
1134 \\
1337 \\
1464 \\
967\end{array}$ & $\begin{array}{r}1320 \\
1033 \\
922 \\
1236 \\
919 \\
1308 \\
1062\end{array}$ & $\begin{array}{r}1240 \\
1085 \\
1144 \\
1236 \\
1137 \\
989 \\
913\end{array}$ & $\begin{array}{r}1050 \\
1192 \\
1017 \\
1006 \\
1195 \\
1012 \\
997\end{array}$ \\
\hline 6 & $\begin{array}{l}\text { BCDA } \\
\text { BDAC } \\
\text { BDCA } \\
\text { CADB } \\
\text { CBDA } \\
\text { DABC } \\
\text { DACB } \\
\text { DBAC } \\
\text { DBCA }\end{array}$ & $\begin{array}{l}1809 \\
1550 \\
1756 \\
1671 \\
1629 \\
1762 \\
1757 \\
1525 \\
1568\end{array}$ & $\begin{array}{l}1673 \\
1586 \\
1600 \\
1941 \\
1780 \\
2004 \\
1534 \\
1742 \\
1821\end{array}$ & $\begin{array}{l}1816 \\
1800 \\
1836 \\
1656 \\
1853 \\
1814 \\
1773 \\
1777 \\
1493\end{array}$ & $\begin{array}{l}1797 \\
1742 \\
2146 \\
2122 \\
2025 \\
1856 \\
1598 \\
1935 \\
2032\end{array}$ & $\begin{array}{r}1230 \\
1041 \\
1184 \\
1250 \\
1001 \\
1050 \\
1044 \\
1085 \\
996\end{array}$ & $\begin{array}{r}1220 \\
1072 \\
987 \\
1070 \\
1186 \\
1179 \\
1180 \\
1151 \\
1253\end{array}$ & $\begin{array}{r}1133 \\
1031 \\
1203 \\
1155 \\
1413 \\
965 \\
1097 \\
1185 \\
994\end{array}$ & $\begin{array}{r}1298 \\
972 \\
1360 \\
1255 \\
1343 \\
1052 \\
1110 \\
1208 \\
1159\end{array}$ \\
\hline 8 & $\begin{array}{l}\text { CDAB } \\
\text { CDBA } \\
\text { DCAB } \\
\text { DCBA }\end{array}$ & $\begin{array}{l}1724 \\
1814 \\
1822 \\
1648\end{array}$ & $\begin{array}{l}1959 \\
1713 \\
1828 \\
1615\end{array}$ & $\begin{array}{l}1867 \\
1694 \\
1979 \\
1592\end{array}$ & $\begin{array}{l}1682 \\
1561 \\
1694 \\
1572\end{array}$ & $\begin{array}{r}974 \\
1080 \\
1166 \\
1090\end{array}$ & $\begin{array}{r}1239 \\
1175 \\
1318 \\
951\end{array}$ & $\begin{array}{l}1217 \\
1175 \\
1191 \\
987\end{array}$ & $\begin{array}{r}1100 \\
993 \\
861 \\
1043\end{array}$ \\
\hline \multicolumn{10}{|c|}{ Percentage of Errors } \\
\hline $\mathbf{0}$ & ABCD & 6.3 & 12.5 & 14.1 & 7.8 & 6.3 & 17.2 & 18.8 & 14.1 \\
\hline 2 & $\begin{array}{l}\text { ABDC } \\
\text { ACBD } \\
\text { BACD }\end{array}$ & $\begin{array}{r}15.7 \\
14.1 \\
9.4\end{array}$ & $\begin{array}{l}14.1 \\
20.3 \\
18.8\end{array}$ & $\begin{array}{r}6.3 \\
17.2 \\
15.7\end{array}$ & $\begin{array}{l}18.8 \\
21.9 \\
14.1\end{array}$ & $\begin{array}{r}11.0 \\
17.2 \\
9.4\end{array}$ & $\begin{array}{r}23.5 \\
21.9 \\
9.4\end{array}$ & $\begin{array}{r}3.2 \\
7.8 \\
14.1\end{array}$ & $\begin{array}{r}7.8 \\
7.8 \\
11.0\end{array}$ \\
\hline 4 & $\begin{array}{l}\text { ACDB } \\
\text { ADBC } \\
\text { ADCB } \\
\text { BADC } \\
\text { BCAD } \\
\text { CABD } \\
\text { CBAD }\end{array}$ & $\begin{array}{l}17.2 \\
11.0 \\
23.2 \\
11.0 \\
11.0 \\
18.8 \\
11.0\end{array}$ & $\begin{array}{r}14.1 \\
17.2 \\
12.0 \\
23.2 \\
4.7 \\
12.5 \\
12.5\end{array}$ & $\begin{array}{r}14.1 \\
9.4 \\
9.4 \\
29.5 \\
7.8 \\
6.3 \\
14.4\end{array}$ & $\begin{array}{r}7.8 \\
12.5 \\
9.4 \\
4.7 \\
17.2 \\
4.7 \\
11.0\end{array}$ & $\begin{array}{r}20.3 \\
7.8 \\
23.2 \\
12.5 \\
9.4 \\
23.5 \\
7.8\end{array}$ & $\begin{array}{r}18.8 \\
6.3 \\
15.7 \\
23.5 \\
9.4 \\
20.3 \\
12.5\end{array}$ & $\begin{array}{r}6.3 \\
7.8 \\
9.4 \\
26.6 \\
17.2 \\
6.3 \\
3.2\end{array}$ & $\begin{array}{r}3.2 \\
12.0 \\
9.4 \\
14.1 \\
11.0 \\
6.3 \\
3.2\end{array}$ \\
\hline 6 & $\begin{array}{l}\text { BCDA } \\
\text { BDAC } \\
\text { BDCA } \\
\text { CADB } \\
\text { CBDA } \\
\text { DABC } \\
\text { DACB } \\
\text { DBAC } \\
\text { DBCA }\end{array}$ & $\begin{array}{l}15.7 \\
12.5 \\
11.0 \\
21.9 \\
12.5 \\
17.2 \\
14.1 \\
12.5 \\
15.7\end{array}$ & $\begin{array}{r}9.4 \\
7.8 \\
14.1 \\
11.0 \\
15.7 \\
25.0 \\
7.8 \\
11.0 \\
18.8\end{array}$ & $\begin{array}{r}15.7 \\
29.5 \\
7.8 \\
11.0 \\
21.9 \\
18.8 \\
11.0 \\
9.4 \\
11.0\end{array}$ & $\begin{array}{r}15.7 \\
21.9 \\
23.5 \\
14.1 \\
21.9 \\
23.5 \\
9.4 \\
15.7 \\
11.0\end{array}$ & $\begin{array}{r}12.5 \\
11.0 \\
9.4 \\
9.4 \\
6.3 \\
11.0 \\
7.8 \\
15.7 \\
3.2\end{array}$ & $\begin{array}{r}17.2 \\
18.8 \\
18.8 \\
12.5 \\
11.0 \\
9.4 \\
9.4 \\
26.6 \\
7.8\end{array}$ & $\begin{array}{r}14.1 \\
34.4 \\
4.7 \\
12.5 \\
23.5 \\
17.2 \\
6.3 \\
23.5 \\
6.3\end{array}$ & $\begin{array}{r}12.5 \\
11.0 \\
20.3 \\
15.7 \\
18.8 \\
25.0 \\
9.4 \\
21.9 \\
12.5\end{array}$ \\
\hline 8 & $\begin{array}{l}\text { CDAB } \\
\text { CDBA } \\
\text { DCAB } \\
\text { DCBA }\end{array}$ & $\begin{array}{r}15.7 \\
7.8 \\
9.4 \\
14.1 \\
\end{array}$ & $\begin{array}{l}11.0 \\
18.8 \\
20.3 \\
11.0 \\
\end{array}$ & $\begin{array}{l}15.7 \\
23.5 \\
18.8 \\
14.1 \\
\end{array}$ & $\begin{array}{r}12.5 \\
25.0 \\
6.3 \\
21.9 \\
\end{array}$ & $\begin{array}{r}15.7 \\
9.4 \\
11.0 \\
7.8 \\
\end{array}$ & $\begin{array}{r}17.2 \\
18.8 \\
12.5 \\
6.3 \\
\end{array}$ & $\begin{array}{r}17.2 \\
14.1 \\
7.8 \\
7.8 \\
\end{array}$ & $\begin{array}{l}7.8 \\
7.8 \\
4.7 \\
6.3 \\
\end{array}$ \\
\hline
\end{tabular}

tation $[F(1,30)=17.1, p<.001]$ (see Table 6). The faster responding with successive presentation apparently was due to subjects' having encoded the first string prior to the time from which the response latency is measured (i.e., from presentation of the second string).
All pair types showed faster RTs for the order task than for the item task $[F \mathrm{~s}(1,60) \geq 5.41, p s<.05]$. The rearranged pairs also showed fewer errors for the order task $[F(1,60)=22.3, p<.001]$, whereas the identical and replacement pairs showed slight, nonsignificant trends 
Table 6

Reaction Times (RT, in Milliseconds) and Percentages of Errors (\%E) for Each Pair Type as a Function of Task and Method of Presentation in Experiment 1

\begin{tabular}{|c|c|c|c|c|c|c|}
\hline \multirow{3}{*}{$\begin{array}{c}\text { Method of } \\
\text { Presentation }\end{array}$} & \multicolumn{6}{|c|}{ Pair Type } \\
\hline & \multicolumn{2}{|c|}{ Identical } & \multicolumn{2}{|c|}{ Rearranged } & \multicolumn{2}{|c|}{ Replacement } \\
\hline & RT & $\% \mathrm{E}$ & RT & $\% \mathrm{E}$ & RT & $\% \mathrm{E}$ \\
\hline \multicolumn{7}{|c|}{ Order Task } \\
\hline Simultaneous & 1078 & 3.1 & 836 & 4.2 & 1072 & 15.2 \\
\hline Successive & 745 & 4.7 & 744 & 8.5 & 809 & 12.9 \\
\hline \multicolumn{7}{|c|}{ Item Task } \\
\hline Simultaneous & 1293 & 2.0 & 1788 & 7.6 & 1730 & 14.3 \\
\hline Successive & 845 & $\underline{2.3}$ & 1197 & 12.3 & 1122 & 12.8 \\
\hline
\end{tabular}

Note-Underlines indicate cells for which the correct response was "same."

toward more errors for the order task than for the item task $[F \mathrm{~s}(1,60) \leq 2.01, p s>.10]$. The relative ease of the order task indicates that the discrimination between identical and rearranged pairs required for this task is easier than the discrimination between rearranged and replacement pairs that is required for the item task.

Finally, RTs for the item task benefited substantially more from successive presentation than did those for the order task, as indicated by the significant task $\times$ method interaction obtained for the rearranged and replacement pairs $\left[F_{\mathrm{S}}(1,60) \geq 6.17\right.$, ps $\left.<.02\right]$, but not for the identical pairs $(F<1.0)$. None of the pair types showed a task $\times$ method interaction for the percentage of errors $(F \mathrm{~s}<1.0)$. This greater benefit of successive presentation for the item task may be an indirect consequence of the slower absolute RTs for that task.

\section{Permutation}

The effects of permutation for the rearranged pairs were of primary interest for evaluating the role of total displacement and the relative serial-position weightings for the order and item tasks. The permutation $\times$ task interaction was highly significant for both RTs and errors $[F \mathrm{~s}(22,1320) \geq 25.5, p s<.001]$, indicating that the permutation effects were different for the two tasks. In general, the tasks showed mirror-image patterns (see Table 3).

The permutation main effects also were significant for both dependent measures $\left[F_{\mathrm{s}}(22,1320) \geq 5.14\right.$, ps $<.001$ ], showing that the patterns for the two tasks were not entirely mirror images. As discussed by Proctor and Healy (1985, p. 528), this finding is illustrated best by negating the scores from one task (e.g., the order task) so that any negative or mirror-image relation between the two tasks is changed to a positive relation. When analyzed in this manner, the terms for the permutation $x$ task interaction and the permutation main effect in the previous unnegated analyses are interchanged, with the main effect indicating the consistent permutation pattern for the tasks and the interaction indicating residual differences that cannot be attributed to the mirror-image relation. Therefore, the previous analyses indicate similar, but not identical, permutation patterns for the order and item tasks when the direction is disregarded.

More specific comparisons of the permutation patterns were conducted to evaluate the extent to which displacement count is the primary determinant of relative difficulty, as predicted by the position-sensitive comparison model, and whether evidence exists for a greater leftto-right weighting of serial positions by subjects who perform the order task. For these comparisons, all main effects and interactions involving permutation refer to analyses performed with the scores from one task negated.

Differences between permutations with different displacement counts. The first set of analyses collapsed the data across the rearranged permutations with equivalent displacement counts, and displacement count $(2,4,6$, or 8 ) then was included as a factor. These data are presented in Table 7. Both RTs and error rates showed main effects of displacement $\left[F_{\mathrm{s}}(3,180) \geq 99.4, p \mathrm{~s}<.001\right]$, indicating that amount of displacement exerted considerable influence. Trend analyses show significant linear $[F \mathrm{~s}(1,60) \geq 123.4, p \mathrm{~s}<.001]$ and quadratic $\left[F_{\mathrm{s}}(1,60) \geq 66.4\right.$, ps $\left.<.001\right]$ components for both RTs and errors, with the RTs also showing a significant cubic trend $[F(1,60)=29.5, p<.001]$. These orthogonal components indicate that the predominant tendency was for RTs and error rates to decrease with increasing displacement for the order task and to increase for the item task, with the difference between adjacent displacement counts decreasing at large displacements. Therefore, total displacement shows a systematic influence on response latencies and errors even for the stronger, less confounded manipulation possible with four-letter strings.

No interactions involving dispacement were significant for RTs $[F \mathrm{~s}(3,180) \leq 2.59, p s>.05]$. Thus, within chance limits, the effects of displacement on RTs were equivalent for the order and item tasks, as well as for simultaneous and successive presentation. However, for the error data, both the displacement $x$ method interaction $[F(3,180)=9.59, p<.001]$ and the displacement $x$ task interaction $[F(3,180)=39.6, p<.001]$ were significant. These interactions indicate that the effect of displacement on errors was larger for successive than for simultaneous presentation, and was larger for the order task than for the item task. Because comparisons between

\section{Table 7}

Reaction Times (RT, in Milliseconds) and Percentages of Errors (\%E) for Rearranged Pairs as a Function of Displacement Count, Task, and Method of Presentation in Experiment 1

\begin{tabular}{|c|c|c|c|c|c|c|c|c|}
\hline \multirow{3}{*}{$\begin{array}{l}\text { Method of } \\
\text { Presentation }\end{array}$} & \multicolumn{8}{|c|}{ Displacement Count } \\
\hline & \multicolumn{2}{|c|}{2} & \multicolumn{2}{|c|}{4} & \multicolumn{2}{|c|}{6} & \multicolumn{2}{|c|}{8} \\
\hline & RT & $\% \mathrm{E}$ & $\overline{\mathrm{RT}}$ & \%E & $\mathbf{R T}$ & $\% \mathrm{E}$ & $\mathbf{R T}$ & $\% \mathrm{E}$ \\
\hline \multicolumn{9}{|c|}{ Order Task } \\
\hline $\begin{array}{l}\text { Simultaneous } \\
\text { Successive }\end{array}$ & $\begin{array}{r}1074 \\
925\end{array}$ & $\begin{array}{l}15.8 \\
29.5\end{array}$ & $\begin{array}{l}906 \\
789\end{array}$ & $\begin{array}{l}3.9 \\
8.6\end{array}$ & $\begin{array}{l}754 \\
683\end{array}$ & $\begin{array}{l}1.5 \\
3.6\end{array}$ & $\begin{array}{l}721 \\
667\end{array}$ & $\begin{array}{l}1.9 \\
1.9\end{array}$ \\
\hline \multicolumn{9}{|c|}{ Item Task } \\
\hline $\begin{array}{l}\text { Simultaneous } \\
\text { Successive }\end{array}$ & $\begin{array}{l}1578 \\
1002 \\
\end{array}$ & $\begin{array}{l}6.4 \\
7.6 \\
\end{array}$ & $\begin{array}{l}1738 \\
1140 \\
\end{array}$ & $\begin{array}{l}6.2 \\
9.5\end{array}$ & $\begin{array}{l}1879 \\
1284 \\
\end{array}$ & $\begin{array}{r}8.6 \\
14.4 \\
\end{array}$ & $\begin{array}{l}1831 \\
1248 \\
\end{array}$ & $\begin{array}{r}8.8 \\
15.3 \\
\end{array}$ \\
\hline
\end{tabular}


positions are pertinent in the item task, but not in the order task, the latter finding is instructive and emphasizes that the between-position comparisons cannot be ignored.

The importance of total displacement is clear when the three alternative characteristics of the rearranged pairs are considered. None of these characteristics-which include (1) the first position in which a mismatch occurs, (2) the total number of within-position mismatches, and (3) the minimum number of perturbations necessary for the string representations to have the same order-relate closely to the relative difficulties of the various pair types. This point can be illustrated by examples using each characteristic. First, the initial mismatch for all of the rearranged pairs, except the ABDC, ACBD, ACDB, ADBC, and ADCB pair types, occurs in the first, or leftmost, position (see Table 1). Yet, as can be seen in Table 3, RTs to the pairs that mismatch in the first position span a broad range for both tasks and systematically follow displacement count (e.g., note the relatively clean separation between RTs to pairs with displacement counts of 4 and 6). Second, the BACD, CBAD, and DBCA pairs are equivalent on the number of within-position matches (two), as well as on the position of initial difference (first), but have total displacement counts of 2,4 , and 6 , respectively. The RTs (and errors, with one exception) to these pairs are inverse, monotonic functions of displacement count for the order and item tasks (see Table 3). Third, the minimum number of perturbations necessary to produce identical orders varies from three to five for the pairs with displacement counts of 6 and from four to six for the pairs with displacement counts of 8 . However, the results showed little difference between the various pair types within each of these two displacement counts (see below and Table 3). Moreover, for each task, product-moment correlations between the number of perturbations and both RTs and errors (mean $|r|=.657$ ) were consistently about .10 less than were the corresponding correlations between displacement count and the same response measures (mean $|r|=.752$ ). Thus, whereas total displacement is a good indicator of relative difficulty, the alternative characteristics are not as good. These results support the positionsensitive comparison model, which stresses displacement, over models that emphasize the alternative characteristics.

Differences between permutations with equivalent displacement counts. The second set of analyses involved comparing permutations that had equivalent displacement counts to determine whether total displacement was the only important factor. Separate analyses were performed for the permutations with displacement counts of 2,4 , 6 , and 8. Differences between permutations that had the same displacement count were limited primarily to those with counts of 2 and 4 . The four pairs with displacement counts of 8 showed no significant effects involving the permutation factor for either RTs or errors $\left[F_{\mathrm{s}}(3,180) \leq\right.$ 2.20 , ps $>.05]$. For the nine permutations with displacement counts of 6 , the permutation main effect $[F(8,480)$ $=2.24, p<.05]$, permutation $\times$ task interaction
$[F(8,480)=3.20, p<.01]$, and permutation $\times$ method interaction $[F(8,480)=2.52, p<.05]$ were significant for RTs, as was the permutation $\times$ task $\times$ method interaction for errors $[F(8,480)=2.32, p<.05]$. However, these were weak effects that accounted for only a small portion of the variance.

Permutations with displacement counts of 2 and 4 showed significant main effects for permutation on both RTs and errors $\left[F_{\mathrm{s}}(2,120) \geq 18.4\right.$ and $F \mathrm{~s}(6,360) \geq 5.80$, ps $<.001$, for displacement counts of 2 and 4 , respectively]. These effects reflect primarily the fact that pairs with a matching letter in the first position (ABDC and ACBD with displacement counts of 2 and ACDB, ADBC, and $A D C B$ with displacement counts of 4) were more difficult than other pairs (BACD with a displacement count of 2 and $B A D C, B C A D, C A B D$, and CBAD with displacement counts of 4) for the order task, but tended to be easier for the item task. Thus, the leftmost position apparently is weighted most heavily in the judgments for both tasks.

Although the order and item tasks generally showed inverse patterns, significant permutation $\times$ task interactions were present in the RT and error-rate data for both the permutations with displacement counts of $2[F \mathrm{~s}(2,120) \geq$ $14.1, p s<.001]$ and those with counts of $4[F \mathrm{~s}(6,360)$ $\geq 2.20, p \mathrm{~s}<.05]$. These interactions indicate that the difference between pairs that had a match in the leftmost position and those that did not was greater for the order task.

Two important points should be emphasized regarding the differences between permutations with equivalent displacement counts. First, for the three-letter strings examined previously (Proctor \& Healy, 1985), only a single comparison (the ACB permutation vs. the BAC permutation) provided evidence relevant to the question of whether the leftmost position was weighted relatively more heavily in the order task than in the item task. Although the results were consistent with this possibility (i.e., the magnitude of the disadvantage for the ACB permutation in the order task was greater than the corresponding advantage for that permutation in the item task), an alternative interpretation is possible: That is, pairs of the ACB type could be particularly difficult in the order task simply because they most closely resemble the $A B C$ pairs, from which they must be discriminated in that task but not in the item task. Because the permutations of fourletter strings that have displacement counts of 4 are not highly similar to the identical pairs, the fact that the order task also showed a greater serial-position effect for these permutations in the present experiment provides strong evidence for a greater left-to-right weighting in that task.

Second, for permutations with displacement counts of 2, pairs of the ACBD type were intermediate in RT and accuracy between pairs of the ABDC type and pairs of the BCAD type for the order task (but not for the item task). Because the ACBD and ABDC permutations differ in whether the fourth or second position contains a match, 
this result provides evidence that the left-to-right decrease in weighting is gradual and is not just a difference between the first position and the remaining ones.

\section{Replacement Pairs}

Order task. Only a subset of the possible replacement pairs (those based on the identical permutation) was used in the order task. For this task, the replacement pairs showed significant main effects for position of replacement on both $R T s$ and errors $[F \mathrm{~s}(3,90) \geq 5.27$, ps $<.01]$. RTs increased monotonically as the position of replacement shifted from left to right; the error data showed a similar relation, with the exception of a deviation for the third position (see Table 4). Therefore, the position effects for the replaced letter generally are consistent with the hypothesis that a left-to-right weighting of serial positions occurs for the order task.

RTs also showed a significant interaction of position with method of presentation $F(3,90)=6.66, p<.001$, indicating that the left-to-right increase in RT was substantially less with successive than with simultaneous presentation, as is found when order is not manipulated (Krueer, 1984; Proctor et al., 1984). This finding also is consistent with the results obtained by Proctor and Healy (1985) for the order task when three-letter strings were used.

Item task. Replacement pairs for the item task were constructed from all permutations. Both RTs and errors showed significant main effects of permutation $[F \mathrm{~s}(23,690) \geq 3.82, p s<.001]$ and also interactions of permutation with position of replacement $[F \mathrm{~s}(69,2070) \geq$ $3.31, p s<.001]$. Therefore, the position effects were not consistent across the permutations (see Table 5). As found by Proctor and Healy (1985) for three-letter strings, essentially all orderings of difficulty as a function of position of replacement were evident across the various base permutations. Most importantly, the item task, unlike the order task, showed no monotonic increase as position of replacement shifted from left to right for the pairs based on the identical permutation (ABCD). This difference between the two tasks for the replacement pairs is consistent with the differences for the rearranged pairs in suggesting that the left-to-right weighting of positions is greater for the order task. Interestingly, method of presentation did not interact with any factor, meaning that within chance limits the patterns of responding were similar for simultaneous and successive presentation. This outcome also stands in contrast to that obtained for the replacement pairs used in the order task.

\section{Summary}

The primary results of Experiment 1 can be summarized as follows. First, as found previously for three-letter strings (Proctor \& Healy, 1985), the patterns of responses to rearranged pairs of four-letter strings were essentially mirror images for the order and item tasks. Within each task, the primary determinant of the relative difficulty for the respective rearranged pairs was the total number of positions by which the letters in one string were displaced to make the second string. Because this evidence was ob- tained from a stringent test, which involved 23 permutations and four levels of displacement, it supports the hypothesis that total displacement, rather than some correlated characteristic, is the most important cause of relative difficulty.

Second, deviations in difficulty between permutations with equivalent displacement counts occurred when the displacement count was small. These deviations reflect primarily a greater weighting of the leftmost position in the pair relative to the other positions, especially for the order task. Furthermore, for permutations with displacement counts of 2, the order task (but not the item task) showed slower and less accurate responding when withinposition matches (i.e., the same letter in corresponding positions of the two strings) occurred in the two leftmost positions rather than in the leftmost and rightmost positions. Also, the order task (but not the item task) showed a left-to-right increase in RTs and errors for replacement pairs as a function of the position of the replaced letter. Both of the latter two results indicate a gradually decreasing left-to-right weighting of positions for the order task that is not apparent for the item task.

Third, although some quantitative differences existed between the patterns of results obtained with simultaneous and successive presentation, the predominant outcome was one of qualitatively (and in most cases, quantitatively) similar permutation and replacement effects for the two methods of presentation. Thus, systematic differences in the permutation effects for the two methods of presentation do not occur even with four-letter strings, for which differences are more likely. The relative lack of effect for presentation method is consistent with our previous conclusion (Proctor \& Healy, 1985) that the rearrangement effects reflect basic representational and comparison operations.

\section{EXPERIMENT 2}

In Experiment 1 and in our previous experiments (Proctor \& Healy, 1985), the proportion of the crucial rearranged pairs was held constant for the order and item tasks. The "same" and "different" response probabilities also were maintained at equal values of .50 for the two tasks to minimize response bias (Proctor \& Rao, 1983; Proctor et al., 1984). Because the rearranged pairs were classified as "different" for the order task but as "same" for the item task, the compositions of the stimulus sets had to differ in other ways. Specifically, for the order task, only a small subset of the possible replacement pairs (those based on the position-identical permutation) were used to complete the "different" response set, and all of the pairs assigned to the "same" response were identical pairs. For the item task, only a subset of the identical pairs was used to complete the set of stimuli assigned to the "same" response, and the "different" response set was composed entirely of replacement pairs constructed from the complete set of base permutations.

This manner of list construction has two drawbacks. First, although the specific rearranged pairs were equated 
for the order and item tasks, the complete set of pairs was not equated. Because the similarity structure of the stimulus set (i.e., the relations between the alternative stimulus pairs) can influence the pattern of results that is obtained (Crist, 1981), the apparent difference between the two tasks in the relative emphasis on left-to-right weightings of positions could be a function of the stimulus sets and not of the task requirements. Second, because of the limited set of replacement pairs used for the order task, it was not possible to compare directly performance on the replacement pairs between the two tasks, nor was it possible to determine the nature of the replacement effects. The replacement pairs should be examined more systematically because they can provide evidence pertaining to the serial weighting functions, as well as to the position-sensitive comparison model. That is, the model predicts functions for the replacement pairs that are not only qualitatively similar for the order and item tasks, but also in the same direction (rather than reversed as for the rearranged pairs), because the replacement pairs are assigned to the equivalent response ("different") in both tasks.

Experiment 2 addressed the drawbacks of the previous experiments by using two lists for both the order and item tasks that included replacement pairs constructed from all of the base permutations. The lists differed in the percentages of identical and replacement pairs. For the $50 \%$ identical list, $50 \%$ of the pairs were identical, $25 \%$ were rearranged, and $25 \%$ were replacement. For the $25 \%$ identical list, half of the identical pairs from the previous list were altered to replacement pairs, making the composition $25 \%$ identical pairs, $25 \%$ rearranged pairs, and $50 \%$ replacement pairs. The significance of this manipulation was that the percentages of same and different pairs according to the relevant criteria were $50 \%$ when the $50 \%$-identical list was used for the order task and when the $25 \%$-identical list was used for the item task. Therefore, a direct comparison could be made between these lists for responses to the replacement pairs, without having response probability confounded with task.

Experiment 2 also allowed evaluation of the possibility that differences in the stimulus-set structure account for the apparent difference in serial position weightings for the order and item tasks because the exact same sets of stimuli were used for both tasks. Therefore, any differences evident between the two tasks in Experiment 2 cannot be attributed to the structure of the stimulus set. In addition, the influence of response probability and response bias could be examined by comparing performance on the two lists within each task.

\section{Method}

\section{Subjects and Design}

Sixty-four subjects participated in the experiment. They were students enrolled in an introductory psychology course at the University of Colorado, Boulder, who were satisfying a course requirement. Type of matching rule (order, item) and list (50\%-identical and $25 \%$-identical) were varied between subjects, making four groups of 16 subjects each. Type of pair (described below) was manipulated within subjects.

\section{Apparatus}

Stimuli were presented on the display screen of a Visual Technology Visual 200 terminal, which uses a P-4 phosphor of the type used in the display screen for Experiment 1 . Timing was controlled by a Digital PDP 11/03-L minicomputer with a programmable realtime clock.

Subjects responded by pressing one of two response buttons that were mounted on a board. Half of the subjects in each condition responded "same" by pressing the left-hand button and "different" by pressing the right-hand button. For the other half of the subjects the pairing was reversed. RTs were recorded to the nearest millisecond.

\section{Stimuli}

Stimuli were pairs of three-letter strings composed from uppercase versions of all consonants of the alphabet (excluding $Y$ ), with all letters used approximately equally often. No letters were repeated within a string or on successive trials. The two strings in each pair were presented centered immediately above and immediately below an asterisk that served as a fixation point. From an approximate viewing distance of $50 \mathrm{~cm}$, the separation between the top and bottom strings was $.74^{\circ}$. Each letter was $.23^{\circ}$ wide and $.46^{\circ}$ high; the separation between letters was $.04^{\circ}$. Thus, each threeletter string was $.77^{\circ}$ wide.

Two different lists were constructed, with each list containing 360 pairs. In a manner similar to that used for Experiment 1, the types of pairs in each list are indicated by using $A B C$ to designate the first string, then referring to the pair by using letters to reflect the relation of the second string to the first. The lists were composed from the three basic pair types used in Experiment 1: (1) identical pairs (ABC pairs); (2) rearranged pairs (ACB, BAC, $\mathrm{BCA}, \mathrm{CAB}$, and CBA); and (3) replacement pairs (ABX, BXC, $\mathrm{XBA}$, etc.). Each of the five possible rearranged pairs was used equally often, as was each of the 18 possible replacement pairs (6 permutations $\times 3$ positions of replacement).

Both lists contained 270 pairs ( 90 identical, 90 rearranged, and 90 replacement) that were equivalent. That is, these strings were composed of the same letters and were in the same positions for the two lists. The only difference between the two lists was in 90 additional filler items. For the $50 \%$-identical list, these items were identical pairs, which thus made the total number of such pairs in the list 180 . For the $25 \%$-identical list, the additional items were replacement pairs, making the total number of replacement pairs 180. This manipulation allowed the percentage of same pairs and the percentage of different pairs (according to the appropriate classification rule) to be an equivalent $50 \%$ when the order rule was used with the $50 \%$-identical list and when the item rule was used with the $25 \%$-identical list. When the order rule was used with the $25 \%$-identical list, the percentages of same and different pairs were $25 \%$ and $75 \%$, respectively, whereas when the item rule was used with the 50\%-identical list, this relation was reversed.

Every 12 consecutive trials in each list included 3 identical pairs, 3 rearranged pairs, 3 replacement pairs, and 3 filler pairs. Every 5 consecutive rearranged pairs included one of each permutation, and every 18 consecutive replacement pairs (in both the experimental and filler sets) included one of each permutation by position combination.

\section{Procedure}

Subjects were instructed as in Experiment 1. After the instructions, a practice block of 12 trials was presented, followed by the test list of 360 trials. For all subjects, the two strings in each pair were presented successively, with the same general procedure as in Experiment 1. There was a short rest after each of the four 90 trial blocks, but the order of blocks was constant across subjects. 
Table 8

Reaction Times (RT, in Milliseconds) and Percentages of Errors (\%E) for Position-Identical and Rearranged Letter Pairs as a Function of Permutation, Task, and List in Experiment 2

\begin{tabular}{|c|c|c|c|c|c|c|c|c|c|c|c|c|}
\hline \multirow[b]{4}{*}{ List } & \multicolumn{12}{|c|}{ Displacement Count } \\
\hline & \multirow{2}{*}{\multicolumn{2}{|c|}{$\frac{0}{A B C}$}} & \multicolumn{4}{|c|}{2} & \multicolumn{6}{|c|}{4} \\
\hline & & & \multicolumn{2}{|c|}{ ACB } & \multicolumn{2}{|c|}{ BAC } & \multicolumn{2}{|c|}{ BCA } & \multicolumn{2}{|c|}{$\mathrm{CAB}$} & \multicolumn{2}{|c|}{ CBA } \\
\hline & $\mathbf{R T}$ & $\% \mathrm{E}$ & RT & $\% \mathrm{E}$ & RT & $\% \mathrm{E}$ & RT & \%E & RT & $\% \mathrm{E}$ & $\mathbf{R T}$ & $\mathscr{\% E}$ \\
\hline \multicolumn{13}{|c|}{ Order Task } \\
\hline $\begin{array}{l}50 \% \text { Identical } \\
25 \% \text { Identical }\end{array}$ & $\begin{array}{l}593 \\
575\end{array}$ & $\begin{array}{l}3.0 \\
3.8\end{array}$ & $\begin{array}{l}789 \\
757\end{array}$ & $\begin{array}{l}17.4 \\
15.6\end{array}$ & $\begin{array}{l}701 \\
653\end{array}$ & $\begin{array}{l}6.6 \\
2.1\end{array}$ & $\begin{array}{l}626 \\
572\end{array}$ & $\begin{array}{l}1.4 \\
0.0\end{array}$ & $\begin{array}{l}677 \\
574\end{array}$ & $\begin{array}{l}2.1 \\
0.3\end{array}$ & $\begin{array}{l}606 \\
569\end{array}$ & $\begin{array}{l}3.1 \\
0.7\end{array}$ \\
\hline \multicolumn{13}{|c|}{ Item Task } \\
\hline $\begin{array}{l}50 \% \text { Identical } \\
25 \% \text { Identical }\end{array}$ & $\begin{array}{l}558 \\
675 \\
\end{array}$ & $\begin{array}{l}0.8 \\
2.4 \\
\end{array}$ & $\begin{array}{l}826 \\
930 \\
\end{array}$ & $\begin{array}{l}2.1 \\
6.3 \\
\end{array}$ & $\begin{array}{r}903 \\
1004 \\
\end{array}$ & $\begin{array}{r}5.9 \\
10.1 \\
\end{array}$ & $\begin{array}{r}995 \\
1154 \\
\end{array}$ & $\begin{array}{r}9.7 \\
14.2 \\
\end{array}$ & $\begin{array}{r}969 \\
1029 \\
\end{array}$ & $\begin{array}{r}12.7 \\
9.7 \\
\end{array}$ & $\begin{array}{r}956 \\
1055\end{array}$ & $\begin{array}{l}9.4 \\
9.7\end{array}$ \\
\hline
\end{tabular}

\section{Results and Discussion}

As in Experiment 1, mean RTs for correct responses and percentages of errors were obtained for each subject as a function of type of pair. These data are summarized in Table 8 for identical and rearranged pairs and in Table 9 for replacement pairs. So that the comparisons between the two lists are based on responses to the same pairs of letter strings, the data for the identical and replacement pairs are based only on the subsets that were common to both lists, not on the filler items. ${ }^{2}$

\section{Task}

As in previous experiments, the order task was easier than the item task (see Table 10). This relation was most apparent for the rearranged pairs and for the replacement pairs, both of which showed faster and more accurate responding in the order task $[F \mathrm{~s}(1,60) \geq 9.52, p \mathrm{~s}<$
.005]. Identical pairs, on the other hand, were less accurate for the order task $[F(1,60)=7.45, p<.01\}$, with the RTs to these pairs showing no overall difference between the two täsks $[F(1,60)=1.28, p>.05]$. The relative lack of difference in difficulty between the tasks for the identical pairs is consistent with the findings of Proctor and Healy (1985) for three-letter strings, although with the four-letter strings used in Experiment 1, RTs for the identical pairs were significantly faster in the order task.

\section{List}

The two lists differed in the percentages of identical and replacement pairs. The $25 \%$-identical list creates a bias toward the "different" response for the order task (i.e., the correct response for $75 \%$ of the pairs is "different"), whereas the $50 \%$-identical list creates a bias toward the "same" response for the item task (i.e., the correct response for $75 \%$ of the pairs is "same").

Table 9

Reaction Times (RT, in Miliseconds) and Percentages of Errors (\%E) for Replacement Pairs as a Function of Task, Permutation, Position of Replacement, and List in Experiment 2

\begin{tabular}{|c|c|c|c|c|c|c|c|c|c|c|c|c|}
\hline \multirow{4}{*}{$\begin{array}{c}\text { List and Position } \\
\text { of Replacement }\end{array}$} & \multicolumn{12}{|c|}{ Displacement Count of Base Permutation } \\
\hline & \multirow{2}{*}{\multicolumn{2}{|c|}{$\frac{0}{\mathrm{ABC}}$}} & \multicolumn{4}{|c|}{2} & \multicolumn{6}{|c|}{4} \\
\hline & & & \multicolumn{2}{|c|}{ ACB } & \multicolumn{2}{|c|}{ BAC } & \multicolumn{2}{|c|}{ BCA } & \multicolumn{2}{|c|}{ CAB } & \multicolumn{2}{|c|}{ CBA } \\
\hline & RT & $\% \mathrm{E}$ & $\mathbf{R T}$ & $\% \mathrm{E}$ & RT & \%E & RT & $\% \mathrm{E}$ & RT & $\% \mathrm{E}$ & RT & $\% \mathrm{E}$ \\
\hline \multicolumn{13}{|c|}{ Order Task } \\
\hline $\begin{array}{c}50 \% \text { Identical } \\
1 \\
2 \\
3\end{array}$ & $\begin{array}{l}616 \\
694 \\
740\end{array}$ & $\begin{array}{l}3.7 \\
3.7 \\
7.5\end{array}$ & $\begin{array}{l}595 \\
617 \\
635\end{array}$ & $\begin{array}{l}2.5 \\
0.0 \\
5.0\end{array}$ & $\begin{array}{l}627 \\
573 \\
573\end{array}$ & $\begin{array}{l}3.7 \\
0.0 \\
0.0\end{array}$ & $\begin{array}{l}602 \\
629 \\
614\end{array}$ & $\begin{array}{l}1.2 \\
5.0 \\
0.0\end{array}$ & $\begin{array}{l}601 \\
577 \\
595\end{array}$ & $\begin{array}{l}3.7 \\
0.0 \\
0.0\end{array}$ & $\begin{array}{l}620 \\
593 \\
661\end{array}$ & $\begin{array}{l}1.2 \\
2.5 \\
7.5\end{array}$ \\
\hline $\begin{array}{c}25 \% \text { Identical } \\
1 \\
2 \\
3\end{array}$ & $\begin{array}{l}540 \\
611 \\
736\end{array}$ & $\begin{array}{l}0.0 \\
0.0 \\
7.5\end{array}$ & $\begin{array}{l}545 \\
567 \\
604\end{array}$ & $\begin{array}{l}0.0 \\
0.0 \\
2.5\end{array}$ & $\begin{array}{l}565 \\
548 \\
521\end{array}$ & $\begin{array}{l}1.2 \\
1.2 \\
0.0\end{array}$ & $\begin{array}{l}533 \\
541 \\
567\end{array}$ & $\begin{array}{l}0.0 \\
0.0 \\
1.2\end{array}$ & $\begin{array}{l}530 \\
542 \\
539\end{array}$ & $\begin{array}{l}0.0 \\
1.2 \\
0.0\end{array}$ & $\begin{array}{l}538 \\
517 \\
546\end{array}$ & $\begin{array}{l}1.2 \\
1.2 \\
1.2\end{array}$ \\
\hline \multicolumn{13}{|c|}{ Item Task } \\
\hline $\begin{array}{c}50 \% \text { Identical } \\
1 \\
2 \\
3\end{array}$ & $\begin{array}{l}789 \\
808 \\
802\end{array}$ & $\begin{array}{l}6.2 \\
5.0 \\
3.7\end{array}$ & $\begin{array}{l}891 \\
777 \\
840\end{array}$ & $\begin{array}{l}7.5 \\
2.5 \\
3.7\end{array}$ & $\begin{array}{l}979 \\
799 \\
903\end{array}$ & $\begin{array}{l}7.5 \\
0.0 \\
6.2\end{array}$ & $\begin{array}{l}791 \\
858 \\
869\end{array}$ & $\begin{array}{l}2.5 \\
2.5 \\
1.2\end{array}$ & $\begin{array}{l}767 \\
767 \\
831\end{array}$ & $\begin{array}{l}7.5 \\
2.5 \\
2.5\end{array}$ & $\begin{array}{l}796 \\
706 \\
824\end{array}$ & $\begin{array}{l}2.5 \\
0.0 \\
3.7\end{array}$ \\
\hline $\begin{array}{c}25 \% \text { Identical } \\
1 \\
2 \\
3\end{array}$ & $\begin{array}{l}875 \\
874 \\
958\end{array}$ & $\begin{array}{l}3.7 \\
3.7 \\
5.0\end{array}$ & $\begin{array}{r}1137 \\
871 \\
910\end{array}$ & $\begin{array}{r}12.5 \\
7.5 \\
0.0\end{array}$ & $\begin{array}{r}1123 \\
1019 \\
962\end{array}$ & $\begin{array}{r}10.0 \\
3.7 \\
3.7\end{array}$ & $\begin{array}{l}912 \\
926 \\
964\end{array}$ & $\begin{array}{l}5.0 \\
1.2 \\
2.5\end{array}$ & $\begin{array}{l}926 \\
868 \\
906\end{array}$ & $\begin{array}{l}3.7 \\
2.5 \\
2.5\end{array}$ & $\begin{array}{l}928 \\
792 \\
995 \\
\end{array}$ & $\begin{array}{l}3.7 \\
1.2 \\
7.5 \\
\end{array}$ \\
\hline
\end{tabular}


All pair types showed task $\times$ list interactions; the interaction was significant on $\mathrm{RTs}\left[F_{\mathrm{s}}(1,60) \geq 4.25\right.$, $p s<.05]$ but not on errors $\left[F_{\mathrm{S}}(1,60) \leq 2.60, p s>.05\right]$ for the identical and replacement pairs, and on errors $[F(1,60)=4.27, p<.05]$ but not on RTs $[F(1,60)=$ $2.31, p>.05]$ for the rearranged pairs. In general, these interactions reflect the fact that responding was faster and more accurate when the list created a response bias (the $25 \%$-identical list for the order task and the $50 \%$-identical list for the item task) than when it did not. This relation was most apparent for the pair types assigned to the predominant response (the rearranged and replacement pairs in the order task; the rearranged and identical pairs in the item task), but it also tended to hold for the pair types assigned to the alternative response (the identical pairs in the order task and the replacement pairs in the item task). Most importantly, no additional terms involving list were significant for either the rearranged or replacement pairs, indicating that similar permutation and position effects were obtained for the two lists. Therefore, the bias introduced by altering the proportions of "same"' and "different" responses had no systematic influence on the permutation and position effects.

\section{Permutation}

With the scores from one task negated so that the permutation effects would be related positively (see Experiment 1), the permutation main effect was significant for both RTs and errors on rearranged pairs $\left[F_{s}(4,240) \geq\right.$ $34.9, p \mathrm{~s}<.001]$. The permutation main effects reflect, in part, decreases in RTs and errors for the order task as the displacement count increased for the rearranged pairs and increases in these measures for the item task (see Table 11).

The permutation $\times$ task interaction was significant for errors $[F(4,240)=5.60, p<.001]$, but not for RTs $[F(4,240)=2.02, p>.05]$, and is due in part to the fact that the difference between the ACB and BAC pairs, which have displacement counts of 2 , is greater for the order task than for the item task. Because the same stimulus sets were used for both tasks, this finding indicates that the greater left-to-right weighting for the order task

\section{Table 10}

Reaction Times (RT, in Milliseconds) and Percentages of Errors (\%E) for Each Pair Type as a Function of Task and List in Experiment 2

\begin{tabular}{|c|c|c|c|c|c|c|}
\hline \multirow[b]{3}{*}{ List } & \multicolumn{6}{|c|}{ Pair Type } \\
\hline & \multicolumn{2}{|c|}{ Identical } & \multicolumn{2}{|c|}{ Rearranged } & \multicolumn{2}{|c|}{ Replacement } \\
\hline & RT & $\% \mathrm{E}$ & RT & $\% \mathrm{E}$ & RT & $\% \mathrm{E}$ \\
\hline \multicolumn{7}{|c|}{ Order Task } \\
\hline $50 \%$ Identical & 593 & 3.0 & 680 & 6.1 & 620 & 2.6 \\
\hline $25 \%$ Identical & $\underline{575}$ & 3.8 & 625 & 3.7 & 561 & 1.0 \\
\hline \multicolumn{7}{|c|}{ Item Task } \\
\hline $50 \%$ Identical & 558 & 0.8 & 930 & 7.9 & 822 & 3.7 \\
\hline $25 \%$ Identical & $\overline{675}$ & $\overline{2.4}$ & $\overline{1034}$ & 10.0 & 941 & 4.4 \\
\hline
\end{tabular}

Note-Underlines indicate cells for which the correct response was "same."
Table 11

Reaction Times (RT, in Milliseconds) and Percentages of Errors (\%E) for Rearranged Letter Pairs as a Function of Displacement Count, Task, and List in Experiment 2

\begin{tabular}{|c|c|c|c|c|}
\hline \multirow[b]{3}{*}{ List } & \multicolumn{4}{|c|}{ Displacement Count } \\
\hline & \multicolumn{2}{|c|}{2} & \multicolumn{2}{|c|}{4} \\
\hline & RT & $\% \mathrm{E}$ & RT & $\% \mathrm{E}$ \\
\hline \multicolumn{5}{|c|}{ Order Task } \\
\hline $50 \%$ Identical & 745 & 12.0 & 636 & 2.2 \\
\hline $25 \%$ Identical & 705 & 8.9 & 572 & 0.3 \\
\hline \multicolumn{5}{|c|}{ Item Task } \\
\hline $50 \%$ Identical & 865 & 4.0 & 973 & 10.6 \\
\hline $25 \%$ Identical & 967 & 8.2 & 1079 & 11.2 \\
\hline
\end{tabular}

is a function of the task requirements and not an artifact of the different stimulus sets that were used in previous experiments.

Although the patterns of RTs and errors generally replicated those obtained by Proctor and Healy (1985), an exception occurred for the permutations with a displacement count of 4 . For example, the CAB permutation was consistently the most difficult of these permutations for the item task in the earlier study, yet it was not here. The failure to obtain a consistent across-experiment pattern of deviations between the permutations with displacement counts of 4 suggests that factors such as the stimulus sets, the specific strings used, and the particular sequences of trials are responsible for the deviations.

\section{Replacement Pairs}

One major purpose of the present experiment was to include a complete set of replacement pairs for both the order and item tasks so that the serial-position functions for these pairs could be compared. Mean RTs and percentages of errors for the replacement pairs are given in Table 9. All analyses were performed without negating the scores from one task because the correct classification for these pairs ("different") is the same in the two tasks. Both measures showed significant main effects for the base permutation $[F \mathrm{~s}(5,300) \geq 2.21, p \mathrm{~s}<.053]$ and for position of the replaced letter $\left[F_{\mathrm{S}}(2,120) \geq 3.72\right.$, $p s<.03$ ]. The former effects generally reflect slower and less accurate responding to replacement pairs whose base strings had displacement counts of $0(M=754 \mathrm{msec}$; percent errors [\%E] $=4.1)$ or $2(M=758 \mathrm{msec} ; \% \mathrm{E}=$ 3.4) than to those whose base displacement count was 4 $(M=716 \mathrm{msec} ; \% \mathrm{E}=2.3)$. The pairs with smaller base displacement counts probably are more difficult because they are more similar to the identical and rearranged pairs from which they must be discriminated. The position main effects reflect an overall U-shaped function in which responses were faster $(M \mathrm{~s}=743,712$, and $754 \mathrm{msec}$ for positions 1,2 , and 3 , respectively) and more accurate (\% $\mathrm{E}$ $=3.8,2.0$, and 3.1 for positions 1,2 , and 3 ) when the replacement was in the second position.

The position and base permutation factors both interacted with task, attaining standard significance levels on 
both RTs and errors for the position $\times$ task interaction $[F \mathrm{~s}(2,120) \geq 4.78$, ps $<.025]$ but only on RTs for the permutation $\times$ task interaction $[F(5,300)=12.8$, $p<.001]$ (for errors, the corresponding $F$ ratio was 2.03, $p<.075)$. The permutation $\times$ task interaction reflects primarily different orderings of difficulty for the two tasks on the pairs constructed from base permutations with counts of 0 and 2. That is, for the order task, responses generally decreased in latency from the $A B C$ to the ACB to the BAC base permutation, whereas for the item task, the relation generally was reversed (see Table 9). The position interactions were due to the fact that only the item task showed the U-shaped functions $(M \mathrm{~s}=$ 910,839 , and $897 \mathrm{msec}$ and $6.0 \%, 2.7 \%$, and $3.5 \%$ for RTs and errors with positions 1,2 , and 3, respectively), with the order task showing a monotonically increasing function for RTs ( $M \mathrm{~s}=576,584$, and $611 \mathrm{msec}$ for positions 1,2 , and 3) and a somewhat similar function for errors $(M \mathrm{~s}=1.5 \%, 1.2 \%$, and $2.7 \%$ for positions 1,2 , and 3). In other words, the order task showed a more direct left-to-right ordering than did the item task.

The only remaining significant terms involving permutation and position were the interactions of those two variables for both $R T s$ and errors $\left[F_{\mathrm{s}}(10,600) \geq 2.22\right.$, $p s<.025]$ and the three-way interaction of those variables with task for the RTs only $[F(10,600)=3.45$, $p<.001$. The former interaction reflects the fact that the specific effect of position of replacement was determined partly by the characteristics of the base string permutation. The latter interaction indicates that the patterns were somewhat different for the two tasks.

To examine the three-way interaction in more detail, separate analyses were performed for each of the six base permutations. These analyses showed that task interacted significantly with position for the ACB permutation on errors $[F(2,120)=4.81, p<.01]$ and for the $A B C$, $A C B$, and CBA permutations on $\operatorname{RTs}\left[F_{\mathrm{s}}(2,120) \geq 5.11\right.$, ps $<.01]$. The interaction evident for the CBA permutation was only a quantitative one, with the order and item tasks showing similar qualitative position effects (see Table 9). Therefore, the only task interactions yielding qualitatively different position effects were those in which the strings for the base permutation matched in the first position (i.e., the $A B C$ and $A C B$ permutations). For these permutations, the order task showed that the pairs increased in difficulty as the position of replacement moved from position 1 to position 3 (i.e., from left to right; see Table 9). For the $A B C$ permutation, the item task showed little influence of position of replacement, particularly between positions 1 and 2, whereas for the ACB permutation, difficulty increased as the position of replacement changed from 2 to 3 to 1 (see Table 9). The stronger leftto-right ordering of difficulty in the order task for the replacement pairs whose base permutations contain a match in the first position is consistent with the greater left-to-right weighting obtained for rearranged pairs.

\section{Summary}

Experiment 2 indicated that the generally mirror-image displacement effects for the order and item tasks were obtained even when the same stimulus sets were used for the two tasks. Moreover, the displacement and replacement effects were unaffected by the proportions of identical and replacement pairs contained in the sets, as well as by the proportions of "same" and "different" classifications, although response bias was affected by these manipulations. Most importantly, though, the relations between the rearranged pairs with displacement counts of 2 (the ACB and BAC permutations) showed a relatively greater weighting for the leftmost position in both lists of the order task, ruling out the possibility that the difference observed in previous experiments was due to the use of different stimulus sets for the order and item tasks or to any consequent differences in response bias.

Consistent with a prediction of the position-sensitive comparison model, the similarity of the order and item tasks extended to the effects of position of replacement. That is, for replacement pairs, the two tasks showed qualitatively similar permutation effects that were not mirror images of each other. Likewise, they showed qualitatively similar position effects for all base permutations except those that contained a within-position match in the leftmost location (the $\mathrm{ABC}$ and $\mathrm{ACB}$ base permutations). For the latter permutations, however, the order task showed a monotonic increase in difficulty as the position of replacement shifted from left to right, whereas the item task showed either a small effect of serial position (the ABC permutation) or a U-shaped function (the ACB permutation). This finding confirms that the relative weighting of serial positions decreases more from left to right for the order task than for the item task.

\section{GENERAL DISCUSSION}

Earlier, we (Proctor \& Healy, 1985) found that responses to rearranged pairs of three-letter strings showed approximately mirror-image effects of displacement for the order and item matching tasks. The present Experiment 1 provided a stronger test of the hypothesis that total displacement is the primary determinant of the relative difficulty for rearranged pairs. In this test we used four-letter strings, rather than the three-letter strings we had used earlier, thus allowing many more permutations for the rearranged pairs ( 23 vs. 5 ) that varied across a wider range of displacement counts (4 vs. 2). Systematic, mirror-image effects of total displacement still were apparent for this wider range of displacement, and alternative characteristics of the permutations did not have as much influence on response latencies and accuracies.

The methods used in prior experiments included only a small subset of replacement pairs (those based on the identical permutation) for the order task. As a consequence, a thorough comparison between this task and the 
item task was not possible for the replacement pairs. The method of Experiment 2 allowed the complete set of replacement pairs to be used for both tasks. The tasks also showed similar patterns of results for these pairs. In this case, however, the patterns were in the same direction (i.e., were not mirror-image relations) for the order and item tasks because the replacement pairs were assigned to the same response ("different") for both tasks. The similar patterns of results for the replacement pairs provide further confirmation of the hypothesis that comparable comparison operations underlie performance on the two tasks.

Although Experiments 1 and 2 showed considerable similarities between the order and item tasks for the displacement and replacement effects, the experiments also showed systematic deviations between the tasks. As we found earlier (Proctor \& Healy, 1985), the order task was considerably easier than the item task. More importantly, though, the tasks showed differences in both the displacement and replacement effects that provided strong evidence for a greater left-to-right weighting of positions in the order task. The rearranged pairs for which the two strings contained the same letter in the leftmost position were more difficult than other rearranged pairs with the same displacement counts for the order task. Although the rearranged pairs with a match in the leftmost position also were easier than the other pairs for the item task, the differences were not as large as they were for the order task. This difference between the two tasks was apparent even when response bias varied and the same stimulus sets were used for both tasks (Experiment 2), ruling out the possibility that it was an artifact of response biases or the separate stimulus sets used for each task in the earlier experiments.

For three-letter strings, the only rearranged permutation with a match in the leftmost position (ACB) has a displacement count of 2 (the smallest displacement possible), and therefore is highly similar to the identical $(A B C)$ permutation. Because the identical and rearranged permutations must be discriminated when order is relevant, the relatively greater difficulty for the ACB permutation in the order task could be a function of its similarity to the identical permutation. The four-letter strings used in Experiment 1, however, allow a similar comparison between permutations with displacement counts of 4 that contain a match in the leftmost position and those that do not. Because this comparison also shows a greater difference between these two types of permutations for the order task than for the item task, even though these pairs are not highly similar to the identical pairs, the difference genuinely seems to reflect a greater weighting of the leftmost position when order is relevant. Furthermore, the four-letter strings permit a comparison for permutations with displacement counts of 2 that contain a match either in the leftmost position and in the position adjacent to it or in the leftmost and rightmost positions.
This comparison shows that the greater weighting of the left side of the stimulus in the order task is not restricted to the leftmost position.

The replacement pairs also showed evidence for a leftto-right weighting in the order task. For replacement pairs based on the identical permutation (ABCD in Experiment 1 and $A B C$ in Experiment 2), the order task showed a monotonic increase in difficulty as the position of replacement shifted from left to right, whereas the item task did not. Experiment 2, which allowed comparison between the two tasks for the complete set of replacement pairs, showed that this systematic deviation in the position effects obtained for the respective tasks involved only the base permutations that contain a match in the leftmost position. At first glance, the smaller serial-position effects in the item task than in the order task might suggest simply that subjects can successfully ignore position in the item task, as instructed. However, the fact that the displacement effects are comparable in magnitude for the two tasks is inconsistent with that simple explanation.

\section{Position-Sensitive Comparison Model}

Earlier, we (Proctor \& Healy, 1985) concluded that the results from the order and item tasks were explained best by a position-sensitive comparison model for which the "same"/" different" decision was based on pooled output. According to the model, all letters in one string are compared to all letters in the other string, with the weightings of the component comparisons being a decreasing function of positional separation or displacement (see also Angiolillo-Bent \& Rips, 1982). We interpreted the equivalent displacement effects obtained for the order task (in which the between-position comparisons are extraneous) and the item task (in which the between-position comparisons are germane) as indicating that the position-sensitive comparisons were an unavoidable consequence of the structure of the string representations. The mirror-image relation between the displacement effects for the two tasks suggested that the "same"'/"different" decision was based on pooled output from,the component comparisons.

The present study corroborates the crucial findings that led us to favor the position-sensitive comparison model. The order and item tasks yielded mirror-image displacement effects of equivalent magnitude with the four-letter strings used in Experiment 1, thus confirming the importance of total displacement over a greater range of displacement and permutations. Moreover, Experiment 2 demonstrated that the mirror-image displacement effects are apparent even when the same stimulus sets are used for the two tasks.

Despite the fact that the order and item tasks show similar mirror-image effects of displacement count, the present experiments show conclusively that the serial-position functions for the tasks are different. Experiments 1 and 2 included several systematic comparisons of serialposition effects for the order and item tasks, and all of 
the comparisons showed evidence of a greater left-to-right weighting of positions in the order task. The stronger, more controlled evidence for task differences in the present study forces us to conclude that different processes are used for the two tasks.

The primary issue, then, is how to reconcile the position-sensitive comparison model to the evidence supporting task-specific control processes. The solution we favor is that the subject can regulate the manner in which the mental representation of the stimulus is scanned or can control the weights given to the different parts of the representation. For example, the subject may choose to scan the representation from left to right or give the greatest weight to the information at the left end of the representation.

The distinct serial-position functions could be fit by adjusting parameters in a mathematical implementation of the model proposed in this paper, or in Ratcliff's (1981) related diffusion model, so that the relative difference in weightings assigned to the positions differs between the order and item tasks. However, such ad hoc settings of the weighting parameters would add little to our understanding of the serial-position functions beyond that shown by the data (see Proctor, 1986, for a more detailed discussion of this issue in fitting models to data). In other words, the important question is not whether existing models can accommodate the task-specific serial-position functions, but why subjects would choose to use left-toright scanning or weighting more in the order task than in the item task. The answer to this question should throw considerable light on the processes involved in multiletter matching.

\section{REFERENCES}

Angrolillo-Bent, J. S., \& RIPS, L. J. (1982). Order information in multiple-element comparison. Joumal of Experimental Psychology: Human Perception \& Performance, 8, 392-406.
BAMBER, D. (1969). Reaction times and error rates for "same-different" judgments of multidimensional stimuli. Perception \& Psychophysics, 6, 169-174.

CrIST, W. B. (1981). Matching performance and the similarity structure of the stimulus set. Joumal of Experimental Psychology: General, 110, 269-296.

Krueger, L. E. (1978). A theory of perceptual matching. Psychological Review, 85, 278-304.

KRUEGER, L. E. (1984). Self-termination in same-different judgments: Multiletter comparison with simultaneous and sequential presentation. Journal of Experimental Psychology: Learning, Memory, \& Cognition, 10, 271-284.

LEE, C., \& EsTEs, W. K. (1981). Item and order information in shortterm memory: Evidence for multilevel perturbation processes. Journal of Experimental Psychology: Human Learning \& Memory, 7, 149-169.

Proctor, R. W. (1986). Response bias, criteria settings, and the fast"same" phenomena: A reply to Ratcliff. Psychological Review, 93, 473-477.

Proctor, R. W., Healy, A. F. (1985). Order-relevant and orderirrelevant decision rules in multiletter matching. Joumal of Experimental Psychology: Learning, Memory, \& Cognition, 11, 519-537.

Proctor, R. W., RAO, K. V. (1983). Evidence that the same-different disparity in letter matching is not attributable to response bias. Perception \& Psychophysics, 34, 72-76.

Proctor, R. W., RAo, K. V., \&urst, P. W. (1984). An examination of response bias in multiletter matching. Perception \& Psychophysics, 35, 464-476.

RATCLFF, R. (1981). A theory of order relations in perceptual matching. Psychological Review, 88, 552-572.

RATCLIFF, R., \& HACKER, M. J. (1981). Speed and accuracy of same and different responses in perceptual matching. Perception \& Psychophysics, 30, 303-307.

\section{NOTES}

1. The task analysis for replacement pairs was accomplished by obtaining a single RT and error percentage for each subject that represented the mean values for all of the types of replacement pairs in the appropriate task.

2. Separate analyses that compared the filler items to the corresponding nonfiller items showed that similar patterns of results were obtained for the two sets.

(Manuscript received January 16, 1987; revision accepted for publication March 4, 1987.) 